\title{
COMPARISON OF NEW GENERIC AND ORIGINAL RAMIPRIL IN PATIENTS WITH ARTERIAL HYPERTENSION AND HIGH CARDIOVASCULAR RISK
}

\author{
S.Yu. Martsevich ${ }^{1,2}$, Yu.V. Lukina1,2*, N.A. Dmitrieva1, O.V. Lerman', S.N. Tolpygina', \\ A.A. Serazhim', V.P. Voronina', M.A. Maksimova', L.Yu. Drozdova', A.V. Zakharova' \\ 'State Research Center for Preventive Medicine of Rosmedtechnology. \\ Petroverigsky per. 10, Moscow, 101990 Russia \\ ${ }^{2}$ Chair of Evidence Based Medicine, Moscow Medical Academy named after I.M.Setchenov. \\ Bolshaya Pirogovskaya ul. 6, Moscow, 119991 Russia
}

Comparison of new generic and original ramipril in patients with arterial hypertension and high cardiovascular risk

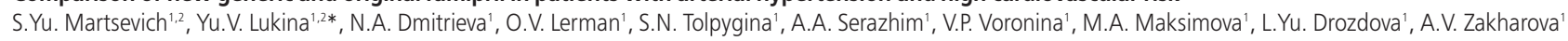
'State Research Center for Preventive Medicine of Rosmedtechnology. Petroverigsky per. 10, Moscow, 101990 Russia

${ }^{2}$ Chair of Evidence Based Medicine, Moscow Medical Academy named after I.M.Setchenov. Bolshaya Pirogovskaya ul. 6, Moscow, 119991 Russia

Aim. To compare efficacy of ramipril generic Hartil ${ }^{\circledast}$ (Egis) to original drug Tritace ${ }^{\circledast}$ in patients with arterial hypertension (HT) of 1 -2 degree and high cardiovascular risk, and to evaluate target blood pressure (BP) achievement when taking each drug separately and in combination with the calcium channel blocker amlodipine.

Material and methods. A total of 27 patients (14 men, 13 women) with HT of 1-2 degree and high risk due to combination of HT with ischemic heart disease, diabetes or previous stroke were included in an open randomized crossover study. Each patient received generic and original ramipril during 6 weeks by turns. Antihypertensive efficacy (the target BP level <130/80) was evaluated every 2 weeks. In case of treatment inefficiency a dose was doubled and amlodipine (Cardilopin, Egis) was added. After 6 weeks of treatment with the first drug of ramipril the second one was administered.

Results. After 6-week Hartil treatment the mean systolic BP (sBP) decreased by 20,0 mm Hg compared to the baseline level, while at Tritace treatment - by $22,2 \mathrm{~mm}$ Hg. The mean diastolic BP (dBP) decreased by 10,8 and $8,6 \mathrm{~mm} \mathrm{Hg}$ respectively (differences between the drugs were insignificant). Twenty patients treated with Hartil and 16 patients treated with Tritace required Cardilopin prescription. The target $\mathrm{BP}<130 / 80$ was achieved in 10 patients $(38,5 \%)$ who took Hartil and in 13 patients (50\%) treated with the original ramipril (differences between the drugs were insignificant).

Conclusion. Therapeutic equivalence of the generic ramipril Hartil and the original drug Tritace was demonstrated. Monotherapy efficacy was low in high-risk patients with $\mathrm{HT}$ of 1-2 degree, and combined therapy with two antihypertensive drugs was effective in $40-50 \%$ of cases.

Key words: arterial hypertension, generic drug, ramipril.

Rational Pharmacother Cardiol 2010;6(1):20-28

\author{
Сравнение нового дженерика и оригинального препарата рамиприла у больных артериальной гипертонией и высоким риском \\ сердечно-сосудистых осложнений \\ С.Ю. Марцевич',2, Ю.В. Лукина,2*, Н.А. Дмитриева', О.В. Лерман', С.Н. Толпыгина', А.А. Серажим', В.П. Воронина', \\ М.А. Максимова', Л.Ю. Дроздова', А.В. Захарова' \\ ${ }^{1}$ Государственный научно-исследовательский центр профилактической медицины Росмедтехнологий. 101990 Москва, Петроверигский пер., 10. \\ ${ }^{2}$ Кафедра доказательной медицины, Московская медицинская академия им. И.М. Сеченова. 119991 Москва, Большая Пироговская ул., 6
}

Цель. Сравнить эффективность дженерического препарата рамиприла Хартил ${ }^{\circledast}$ (Эгис) и оригинального препарата Тритаце ${ }^{\circledast}$ у больных артериальной гипертонией (АГ) 1-2-й степени с высоким сердечно-сосудистым риском и изучить возможность достижения целевого уровня артериального давления (АД) при отдельном приеме каждого препарата и в комбинации с антагонистом кальциевых каналов амлодипином.

Материал и методы. В открытое, рандомизированное, перекрестное исследование включены 27 пациентов (14 мужчин и 13 женщин) с АГ 1-2-й степени с высо ким риском, обусловленным сочетанием АГ с ишемической болезнью сердца, сахарным диабетом, мозговым инсультом (в анамнезе). Каждый пациент поочередно получал в течение 6 недель дженерический и оригинальный препарат рамиприла. Антигипертензивная эффективность (целевой уровень АД <130/80 мм рт.ст.) оценивалась каждые 2 недели. При неэффективности терапии дозу удваивали и добавляли амлодипин (Кардилопин, Эгис). Через 6 недель лечения первым препаратом проводили лечение вторым препаратом рамиприла.

Результаты. В результате 6-недельного лечения Хартилом средний уровень систолического АД (САД) снизился на 20,0 мм рт.ст. по сравнению с исходным, после 6-недельной терапии Тритаце - на 22,2 мм рт.ст. Средний уровень диастолического АД (ДАД) снизился на 10,8 и 8,6 мм рт.ст., соответственно (различия между препаратами незначимы). Назначение Кардилопина потребовалось 20 пациентам при лечении Хартилом и 16 - при терапии Тритаце. Достичь целевого уровня АД <130/80 мм рт.ст. удалось у 10 больных (38,5\%) при лечении Хартилом и у 13 больных (50\%) при использовании оригинального рамиприла (различия между препаратами незначимы).

Заключение. Показана терапевтическая эквивалентность дженерического препарата рамиприла Хартила и оригинального препарата рамиприла Тритаце. У пациентов с АГ 1-2-й степени с высоким риском выявлена низкая эффективность монотерапии, а комбинированная терапия двумя антигипертензивными препаратами эффективна в $40-50 \%$ случаев.

Ключевые слова: артериальная гипертония, дженерический препарат, рамиприл.

РФК 2010;6(1):20-28

*Автор, ответственный за переписку (Corresponding author): jlukina@gnicpm.ru

\section{Сведения об авторах:}

Марцевич Сергей Юрьевич, Д.м.н., профессор, руководитель отдела профилактической фармакотерапии ГНИЦ ПМ, профессор кафедры доказательной медицины ММА

Лукина Юлия Владимировна, К.м.Н., Ст.н.с. отдела профилактической фармакотерапии ГНИЦ ПМ, ассистент кафедры доказательной медицины ММА

Дмитриева Надежда Анатольевна, К.М.Н., Н.С. отдела профилактической фармакотерапии ГНИЦ ПМ
Лерман Ольга Викторовна, к.м.Н., ст.н.с. того же отдела Толпыгина Светлана Николаевна К.М.Н., вед.Н.С. того же отдела Серажим Анна Аркадьевна, К.м.н., Н.С. того же отдела Воронина Виктория Петровна, К.М.Н., Н.С. того же отдела Максимова Марина Александровна, мл.н.С. того же отдела Дроздова Любовь Юрьевна, мЛ.н.с. того же отдела Захарова Алла Витальевна, К.М.Н., Н.С. того же отдела 
The problem of choice of effective and safe drug still remains topical in practical medicine. Nowadays all drugs are divided into two groups: original and generic (copied). Generics make it possible to decrease treatment costs significantly. Due to lower cost generics are available to more number of patients. That is why generic sales increase all over the world. To be registered in Russia equivalence of a generic to an original drug by its quantitative and qualitative structure, physical and chemical properties and equivalence of pharmacokinetic must be estimated. Deviations of these parameters permitted by the Russian legislation lead to registration of drugs with the same international unlicensed name as an original one but with significantly discrepant therapeutic efficacy and safety. That is why comparative studies of new generics and original drugs are of great importance.

Angiotensin converting enzyme (ACE) inhibitors are the first line drugs in patients with arterial hypertension (HT). However, physicians prescribe ACE inhibitor ramipril significantly more rarely than other drugs of the same class, while evidential base of the original ramipril surpasses that of many other ACE inhibitors. A large number of trials confirms efficacy and high safety of the drug in treatment of patients with HT (CARE, DIABHYCAR, DIAL, RACE) [1 -4], chronic heart failure (AIREX, CHARMAdded) $[5,6]$, ischemic heart disease (IHD) (AIRE, APRES, HEART, ONTARGET) [7-10] renal diseases (AASK, REIN) [11-13].

The original ramipril in Russia is Tritace (Sanofi-Aventis, France). Several generics of ramipril were launched during the last few years, one of them is Hartil (Egis, Hungary). Comparative study of generic and original ramipril is relevant. Bioequivalence of the original and a generic drug, which is required for registration, must be confirmed in clinical study of generic efficacy and safety.

The aim of our study was to evaluate efficacy of the new ramipril generic Hartil ${ }^{\circledR}$ in comparison with the original product - Tritace ${ }^{\circledR}$ in patients with essential HT of 1-2 degree and high cardiovascular risk. Target blood pressure (BP) achievement when taking each drug separately or in combination with calcium channel blocker amlodipine (if necessary) was studied as well.

\section{Material and methods}

\section{Patients}

Patients with HT of $1-2$ degree: $140<\mathrm{sBP}<180 \mathrm{~mm} \mathrm{Hg}$ and/or $90<\mathrm{dBP}<110 \mathrm{~mm} \mathrm{Hg}$ and high risk of cardiovascular complications were selected for the study (sBP - systolic BP, dBP - diastolic BP). High cardiovascular risk was determined by combination of $\mathrm{HT}$ with IHD, diabetes, stroke (in history). Patients with secondary HT; severe chronic heart failure (NYHA III-IV); valvular heart diseases; arterial hypotension; myocardial infarction; severe liver and kidney diseases; unstable angina; severe concomitant diseases that
Проблема выбора эффективного и безопасного препарата до сих пор остается актуальной в практической медицине. Все современные препараты подразделяются на две группы - оригинальные и дженерические (копированные). Использование дженериков позволяет значительно снизить затраты на лечение. В результате более низкой стоимости дженерики доступны большему числу пациентов, поэтому объемы их продаж растут во всем мире. Для регистрации дженериков в России обязательна оценка соответствия количественного и качественного состава, физико-химических свойств и фармакокинетической эквивалентности оригинальному препарату. Допускаемые российским законодательством отклонения по этим параметрам приводят к регистрации лекарственных препаратов, имеющих одинаковое международное непатентованное название с оригиналом, однако существенно отличающихся по показателям терапевтической эффективности. Вот почему так важны исследования, посвященные сравнительному изучению клинической эффективности и безопасности новых дженериков и оригинальных препаратов.

Ингибиторы ангиотензин-превращающего фермента (АПФ) в нашей стране занимают первое место по частоте назначения врачами общей практики при лечении пациентов с артериальной гипертонией (АГ). Однако ингибитор АПФ рамиприл назначается существенно реже по сравнению с другими представителями этого класса, хотя доказательная база оригинального рамиприла значительно превосходит таковую у многих других ингибиторов АПФ. Результатами многочисленных исследований подтверждены эффективность и высокая безопасность препарата при лечении пациентов С AГ (CARE, DIABHYCAR, DIAL, RACE) [1-4], хронической сердечной недостаточностью (AIREX, CHARM-Added) [5,6], ишемической болезнью сердца (ИБС) (AIRE, APRES, HEART, ONTARGET) [7-10] и при заболеваниях почек (AASK, REIN) [11-13].

Оригинальным препаратом рамиприла на российском рынке является препарат Тритаце (Sanofi-Aventis, Франция). В последние годы было выпущено несколько дженерических препаратов рамиприла, одним из которых является препарат Хартил (Egis, Венгрия). Выполнение сравнительного исследования с оригинальным препаратом рамиприла представляется актуальным. Биоэквивалентность оригинального и дженерического препаратов, необходимая для регистрации, нуждается в клинических подтверждениях эффективности и безопасности нового препарата.

Цель исследования - изучение эффективности нового дженерического препарата рамиприла Хартила ${ }^{\circledR}$ В сравнении с оригинальным препаратом Тритаце ${ }^{\circledR}$ у больных эссенциальной АГ 1-2-й степени с высоким сердечнососудистым риском. Также изучалась возможность достижения целевого уровня артериального давления (АД) при отдельном приеме каждого препарата и в ком- 


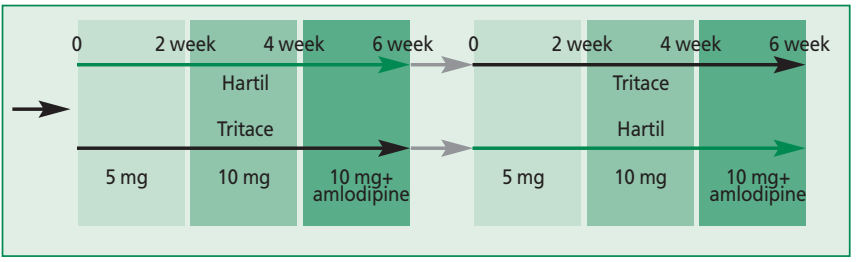

Figure 1. Design of the study

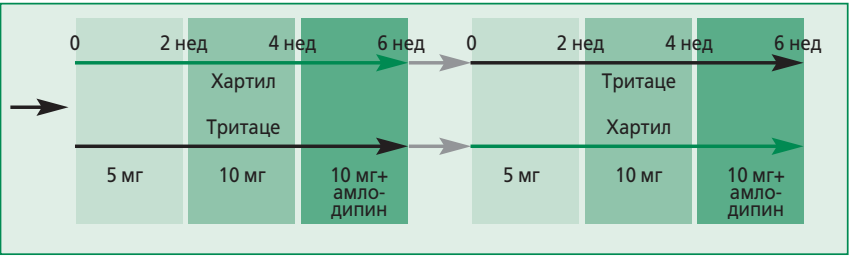

Рисунок 1. Схема исследования

demand permanent taking of drugs that influence BP, atrioventricular block of II-III degree; sinoatrial block; bradycardia (heart rate $<50$ beats per min); bronchial asthma; severe peripheral arterial disorders; high sensitivity to any component of examined medicines were not included into the study.

\section{Design of the study}

This was an open, randomized, crossover, comparative study. The study protocol was approved by the Ethical committee of the State Research Center for Preventive Medicine. Each patient gave written informed consent to participate in the trial. Fig. 1 shows the plan of the study.

Each patient, that participated in the study received Hartil and Tritace one after another. Succession of drug administration was randomized. Wash out period of 7 days during which patients were not receiving any antihypertensive drugs preceded treatment with each drug. The exception was made for $\beta$-blockers in patients after myocardial infarction (in this case the dose was stable during the whole study). In other patients $\beta$-blockers were terminated according to the scheme with the following 7-day wash out period. Nitrates in patients with IHD, and "vascular" therapy in stable doses in patients with previous stroke were allowed as well.

Duration of each drug treatment was 6 weeks. Treatment efficacy evaluation and dose correction were made every 2 weeks. If the treatment was not efficient during first two weeks the dose was doubled. According to clinical guidelines of the European Society of Cardiology the target $\mathrm{BP}$ for hypertensive patients with high risk is below $130 / 80 \mathrm{~mm} \mathrm{Hg}$ [14]. If the target BP $(<130 / 80 \mathrm{~mm} \mathrm{Hg})$ was not achieved after 4-week treatment with ramipril, amlodipine was added (Cardilopin, Egis, Hungary).

The drugs were administered once a day in the morning. The initial dose of ramipril was $5 \mathrm{mg} / \mathrm{d}$. Amlodipine was prescribed in dose of $5 \mathrm{mg}$ a day. BP (in sitting posi- бинации с антагонистом кальциевых каналов амлодипином (при необходимости)

\section{Материал и методы \\ Пациенты}

Для исследования были отобраны пациенты с АГ 1 - 2 степени: $140<$ САД<180 мм рт.ст. и/или 90<дАД<110 мм рт.ст. (сАД - систолическое АД, дАД - диастолическое АД) и высоким риском сердечно-сосудистых осложнений. Последний был обусловлен сочетанием АГ с ИБС, сахарным диабетом (СД), мозговым инсультом (в анамнезе). В исследование не включали больных симптоматической АГ; тяжелой хронической сердечной недостаточностью (NYHA III-IV класс); с пороками сердца; артериальной гипотонией; инфарктом миокарда; тяжелым поражением печени, почек; нестабильной стенокардией; с серьезными сопутствующими заболеваниями, требующими постоянного приема препаратов, оказывающих влияние на АД; атриовентрикулярной блокадой II-III степени; синоаурикулярной блокадой; брадикардией (частота сердечных сокращений (ЧСС) < 50 в мин); бронхиальной астмой; выраженными нарушениями периферического кровообращения; с повышенной чувствительностью к любому из компонентов применяемых препаратов.

\section{Дизайн исследования}

Открытое, рандомизированное, перекрестное, сравнительное исследование. Протокол исследования был одобрен Этическим комитетом Государственного научно-исследовательского центра профилактической медицины. Каждый пациент давал письменное информированное согласие на участие в исследовании. Схема исследования представлена на рис. 1.

Всем пациентам, принимавшим участие в исследовании, поочередно назначали терапию Хартилом и Тритаце. Последовательность назначения препаратов для каждого пациента определялась путем рандомизации. Лечению каждым препаратом предшествовал отмывочный период продолжительностью 7 дней, в течение которого пациенты не принимали антигипертензивные препараты. Исключение составляли бета-адреноблокаторы у пациентов, перенесших инфаркт миокарда (в таких случаях бета-адреноблокатор применялся в стабильной дозе в течение всего времени участия больного в исследовании). При терапии бета-адреноблокаторами у остальных пациентов препарат отменялся по схеме, а далее назначали 7-дневный отмывочный период. Допускалось использование в стабильных дозах на протяжении всего исследования нитратов у больных ИБС и «сосудистой» терапии у больных, перенесших инсульт.

Длительность лечения каждым препаратом составляла 6 недель. Контроль эффективности и корректировку дозы осуществляли каждые 2 недели. При недостаточной эффективности препарата в течение первых 2 недель лечения дозу удваивали. Целевой уровень АД для па- 
tion three times with a 2-minute interval), heart rate and BP target level achievement were evaluated at the beginning of the study and after 2, 4 and 6 weeks of treatment with each drug. Side effects of the therapy were registered when a patient visited physician, treatment compliance was determined.

Antihypertensive therapy was stopped for 7 days after 6 weeks of treatment with the first randomized drug. Then the other drug of ramipril was administered, and the treatment was conducted according to the same scheme.

ECG was registered at the beginning and at the end of the study.

\section{Individual efficacy analysis}

Antihypertensive effect of the studied drugs was evaluated by BP level (change from the base level after 6 -week treatment). The drug was considered effective at BP below $130 / 80 \mathrm{~m} \mathrm{Hg}$ (target BP achievement in HT patients with high risk).

\section{Statistical analysis}

Data were processed with the program package STATISTICA 6. Standard methods of descriptive statistics were applied (means and standard deviations calculation). Parametric (Student's t-test) and nonparametric criteria $\left(\chi^{2}\right.$, Fisher's exact test) were used for data comparison. Data are presented as $\mathrm{M} \pm \sigma(\mathrm{M}$ - mean, $\sigma$ - standard deviation). Differences were considered significant at $\mathrm{p}<0,05$.

\section{Results}

A total of 30 patients were included in the study, but 3 patients withdrew their consents after the screening visit, before randomization, so final analysis included $27 \mathrm{pa}$ tients. Among those - 14 men (52\%) and 13 women (48\%) aged $45-83$ years, the mean age was $65,8 \pm 9,0$ years old. HT duration ranged from 1 to 40 years with the mean value of $17,3 \pm 11,5$ years. All the patients had HT of 1 - 2 degree: 22 patients - degree 1 and 5 patients - degree 2 . Sixteen patients had IHD ( six of them had previous myocardial infarction and received $\beta$-blockers in doses fixed at the first visit), 6 patients had type 2 diabetes, combination of IHD and type 2 diabetes was registered in 4 patients, and 1 patient had previous stroke.

Twenty five patients of 27 , included in the statistical analysis, finished the therapy with both drugs entirely. Two patients completed 6-week treatment with only one drug. One of them received Hartil and quitted the study because of a side effect development. The second one completed the course of Tritace treatment and refused to further participation in the study for the reason not connected with the drug therapy. Thus, 26 patients with HT and high risk of cardiovascular complications received 6-week Hartil treatment, and also 26 patients - treatment with Tritace. циентов с АГ высокого риска по данным клинических рекомендаций Европейского общества кардиологов определен ниже 130/80 мм рт.ст. [14]. При недостижении целевого уровня АД (<130/80 мм рт.ст.) при приеме препаратов рамиприла в течение 4 нед к терапии добавлялся амлодипин (Кардилопин, Egis, Венгрия).

Препараты назначали 1 раз в день утром. Начальная доза рамиприла составляла 5 мг/сут. Амлодипин применяли в дозе 5 мг/сут. Оценку АД (в положении пациента сидя, трижды, с интервалом в 2 мин), ЧСС и достижения целевого АД проводили исходно, а также через 2, 4 и 6 недель терапии каждым препаратом. Во время визитов к врачу регистрировали побочные эффекты проводимой терапии, определяли приверженность лечению.

После 6 недель лечения первым рандомизационным препаратом проводили отмену антигипертензивной терапии на 7 дней. Далее назначался второй препарат рамиприла и лечение проводилось по такой же схеме.

В начале и конце исследования больным регистрировали ЭКГ.

Индивидуальный анализ эффективности

Антигипертензивный эффект препаратов оценивали по результатам измерения АД (изменение от базового уровня после 6-недельной терапии). Препарат считали эффективным, если регистрировали снижение АД ниже уровня 130/80 мм рт.ст. (достижение целевого уровня АД у больных АГ высокого риска).

Статистический анализ

При обработке результатов использовали пакет статистических программ STATISTICA 6.0. Применяли стандартные методы описательной статистики (вычисление средних, средних квадратичных отклонений), для сравнения данных использовали параметрические (парный t-критерий Стьюдента) и непараметрические критерии ( $\chi^{2}$, точный критерий Фишера). Данные представлены в виде $\mathrm{M} \pm \sigma(\mathrm{M}$ - среднее, $\sigma$ - среднее квадратичное отклонение). Различия расценивали как значимые при $p<0,05$.

\section{Результаты}

Всего в исследование было включено 30 пациентов, однако 3 пациента отозвали согласия после визита скрининга, до рандомизации, поэтому в окончательный анализ были включены данные 27 больных. Среди них 14 мужчин (52\%) и 13 женщин (48\%) в возрасте от 45 до 83 лет, средний возраст 65,8土9,0 лет. Длительность АГ колебалась от 1 года до 40 лет и составила в среднем 17,3 11,5 лет. Все пациенты страдали АГ 1-2-й степени: 22 человека - 1 -й степени и 5 больных - 2-й степени. у 16 пациентов высокий риск был обусловлен наличием ИБС (6 человек перенесли инфаркт миокарда и в течение всего времени исследования продолжали прием бета-адреноблокаторов в дозах, зафиксированных на визите включения) , у 6 - наличием СД 2-го типа, 4 па- 
Table 1. Baseline characteristics of the groups

\begin{tabular}{|c|c|c|c|}
\hline Variable & Hartil, $n=13$ & Tritace, $n=14$ & $\mathrm{p}$ \\
\hline Age, years $(M \pm \sigma)$ & $66,0 \pm 9,6$ & $66,7 \pm 8,6$ & 0,15 \\
\hline Height, $\mathrm{cm}(\mathrm{M} \pm \sigma)$ & $167,1 \pm 9,3$ & $169,6 \pm 7,7$ & 0,85 \\
\hline Weight, $\mathrm{kg}(\mathrm{M} \pm \sigma)$ & $82,1 \pm 13,4$ & $79,6 \pm 15,8$ & 0,85 \\
\hline HT duration, years $(M \pm \sigma)$ & $17,7 \pm 9,2$ & $15,8 \pm 11,5$ & 0,33 \\
\hline$s B P, m m ~ H g(M \pm \sigma)$ & $156,2 \pm 8,4$ & $150,3 \pm 7,9$ & 0,36 \\
\hline $\mathrm{dBP}, \mathrm{mm} \mathrm{Hg}(\mathrm{M} \pm \sigma)$ & $89,5 \pm 8,8$ & $87,5 \pm 9,5$ & 0,84 \\
\hline Heart rate, beats per $\min (\mathrm{M} \pm \sigma)$ & $73,5 \pm 5,4$ & $68,4 \pm 10,2$ & 0,08 \\
\hline $\begin{array}{l}\text { IHD, } \mathrm{n} \\
\text { including patients with previous } \\
\mathrm{MI} \text { and } \beta \text {-blockers receiving }\end{array}$ & $\begin{array}{l}7 \\
3\end{array}$ & $\begin{array}{l}9 \\
3\end{array}$ & $\begin{array}{l}\text { NS } \\
\text { NS }\end{array}$ \\
\hline IHD + type 2 diabetes, $n$ & 2 & 2 & NS \\
\hline Type 2 diabetes, $n$ & 3 & 3 & NS \\
\hline Previous stroke, $\mathrm{n}$ & 1 & 0 & NS \\
\hline
\end{tabular}

Таблица 1. Исходная характеристика групп терапии

\begin{tabular}{|c|c|c|c|}
\hline Показатель & Хартил (n=13) & Тритаце (n=14) & $p$ \\
\hline Возраст, лет $(\mathrm{M} \pm \sigma)$ & $66,0 \pm 9,6$ & $66,7 \pm 8,6$ & 0,15 \\
\hline Рост, см $(\mathrm{M} \pm \sigma)$ & $167,1 \pm 9,3$ & $169,6 \pm 7,7$ & 0,85 \\
\hline Bec, кг $(\mathrm{M} \pm \sigma)$ & $82,1 \pm 13,4$ & $79,6 \pm 15,8$ & 0,85 \\
\hline Длительность АГ, лет (M $\pm \sigma)$ & $17,7 \pm 9,2$ & $15,8 \pm 11,5$ & 0,33 \\
\hline сАД, мм рт.ст. (М $\pm \sigma)$ & $156,2 \pm 8,4$ & $150,3 \pm 7,9$ & 0,36 \\
\hline дАД, мм рт.ст. (М $\pm \sigma)$ & $89,5 \pm 8,8$ & $87,5 \pm 9,5$ & 0,84 \\
\hline ЧСС, в мин $(\mathrm{M} \pm \sigma)$ & $73,5 \pm 5,4$ & $68,4 \pm 10,2$ & 0,08 \\
\hline ИБС, $\mathrm{n}$ & 7 & 9 & NS \\
\hline $\begin{array}{l}\text { В т.ч. перенесших ИМ } \\
\text { и принимающих ББ }\end{array}$ & 3 & 3 & NS \\
\hline ИБС+СД 2-го типа, n & 2 & 2 & NS \\
\hline СД 2-го типа, n & 3 & 3 & NS \\
\hline Инсульт в анамнезе, n & 1 & 0 & NS \\
\hline
\end{tabular}

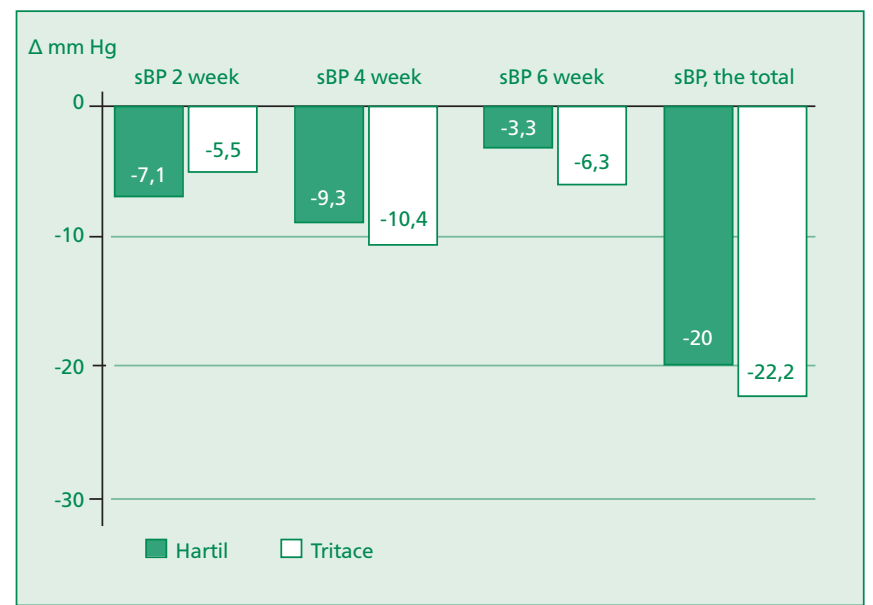

Figure 2. sBP change in the course of the treatment $(\triangle \mathrm{SBP})$ циента имели сочетание ИБС с СД 2-го типа, 1 пациент перенес мозговой инсульт.

Из 27 человек, данные которых были включены в статистический анализ, полностью завершили терапию обоими препаратами 25 пациентов. 2 человека завершили 6-недельное лечение только одним препаратом. Один пациент получал терапию Хартилом и выбыл в связи с развившимся побочным эффектом. Второй пациент завершил курс лечения Тритаце и выбыл вследствие отказа от дальнейшего участия в исследовании по причине, не связанной с приемом исследуемого препарата. Таким образом, 6-недельную терапию Хартилом получили 26 человек и Тритаце - также 26 пациентов с АГ и высоким риском развития осложнений.

В результате рандомизации были сформированы две группы пациентов, в одной из которых первым получаемым препаратом был Хартил, а в другой группе - Тритаце. Значимых отличий по возрасту, заболеваниям, обусловливающим высокий риск, антропометрическим данным и гемодинамическим параметрам между группами выявлено не было (табл. 1).

Оба исследуемых препарата оказывали умеренный антигипертензивный эффект, усиливающийся на фоне комбинированной терапии. Значимых различий между препаратами по влиянию на уровень АД и ЧСС выявлено не было. На рис. 2 и 3 показана динамика сАД и дАД на фоне лечения каждым из препаратов по сравнению с исходными данными.

\section{Клиническая эффективность (индивидуальный анализ)}

По данным индивидуального анализа, целевой уровень АД был достигнут при приеме оригинального рамиприла (Тритаце) в дозе 5 мг/сут у 3 пациентов. Удвоение дозы Тритаце потребовалось 23 пациентам, а еще 16 пациентам для достижения целевого уровня АД к терапии Тритаце добавили 5 мг Кардилопина. При лечении Хартилом первая доза препарата 5 мг/сут у паци-

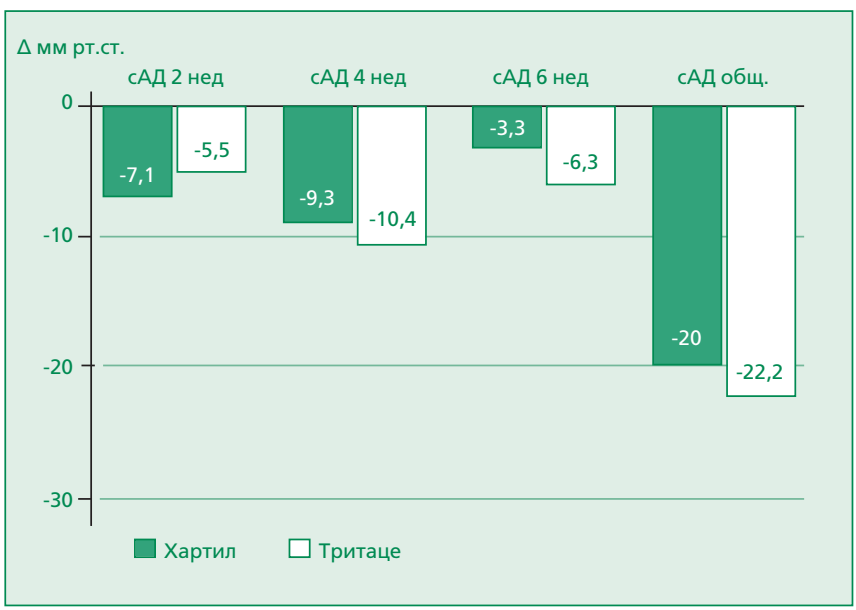

Рисунок 2. Изменение показателей сАД в результате терапии ( $\triangle$ сАД) 


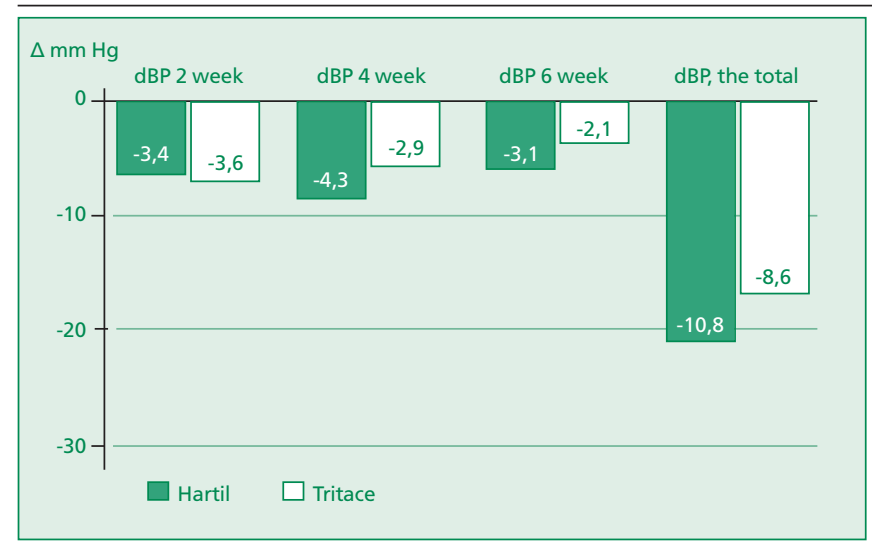

Figure 3. dBP change in the course of the treatment $(\triangle \mathrm{dBP})$

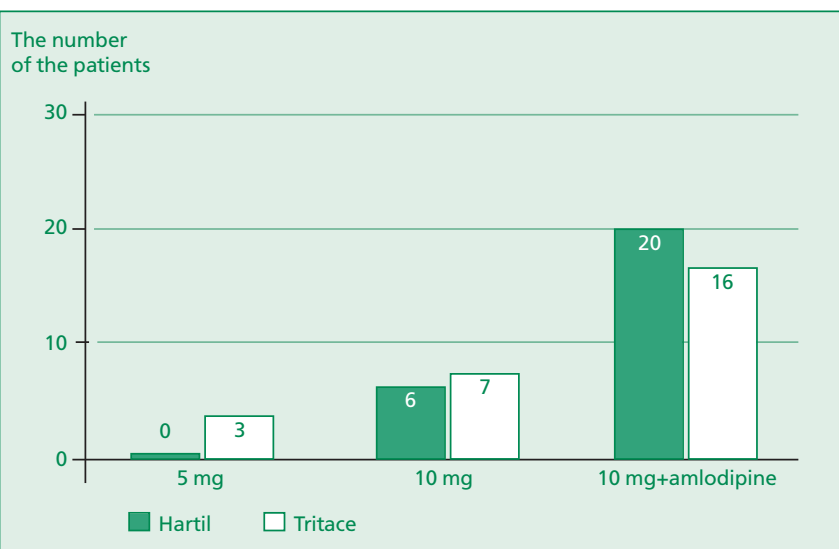

Figure 4. Distribution of the patients depending on a receiving dose and additional amlodipine treatment

Patients were randomized into two groups: the patients of the first group initially received Hartil, while the patients of the other group - Tritace. No significant differences in age, anthropometric data, hemodynamic parameters and diseases determining high risk were revealed in the groups (Table 1).

Both drugs had moderate antihypertensive effect, which increased at combined therapy. There were no significant differences between the drugs concerning $\mathrm{BP}$ level and heart rate. Fig. 2 and 3 show sBP and dBP dynamics compared to baseline at treatment with each drug.

\section{Clinical efficacy (individual analysis)}

According to individual analysis, target BP level at $5 \mathrm{mg}$ of original ramipril (Tritace) was achieved in 3 patients. Twenty three patients required doubling the dose, and $5 \mathrm{mg}$ of Cardilopin was added for 16 patients. Hartil in the initial dose ( $5 \mathrm{mg}$ ) was ineffective in high-risk patients, and dose doubling was necessary for all 26 patients. At that, 20 patients besides dose doubling required Cardilopin administration (Fig.4).

Target BP was reached in 10 patients, who received Hartil and in 13 patients, treated with Tritace. Difference between the drugs was not significant (at comparison of rel-

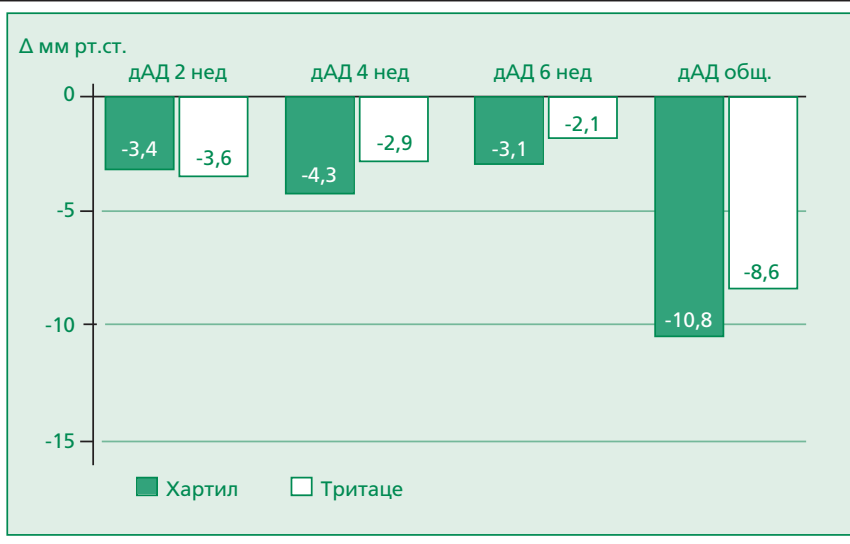

Рисунок 3. Изменение показателей дАД в результате терапии ( $\triangle$ дАД)

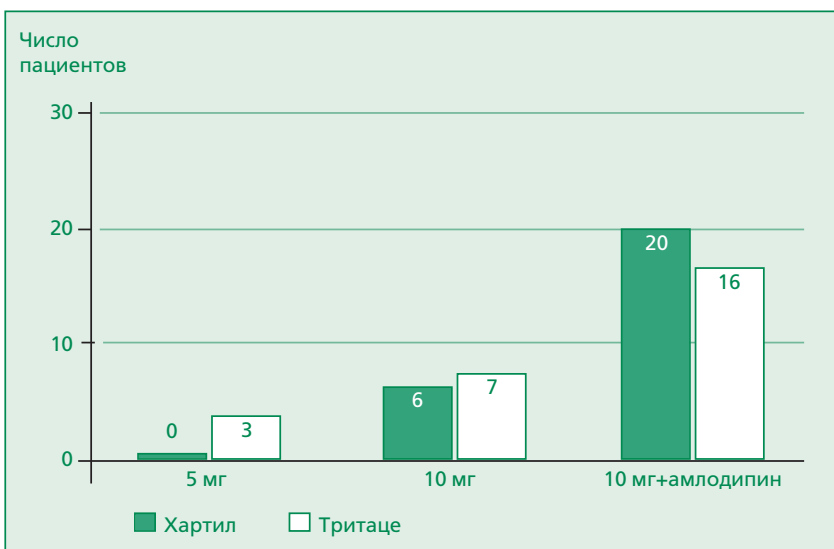

Рисунок 4. Распределение пациентов в зависимости от получаемой дозы и дополнительной терапии амлодипином

ентов высокого риска оказалась неэффективной, и удвоение дозы потребовалось всем 26 пациентам, а 20 пациентам, помимо удвоения дозы препарата, понадобилось назначение Кардилопина (рис. 4).

Целевого уровня АД удалось достичь у 10 пациентов на терапии Хартилом и у 13 - при лечении Тритаце. Различие между препаратами не достигает значимости (при сравнении относительных частот в двух группах критерий $\left.\chi^{2}=0,7, p=0,4\right)$. В табл. 2 указано количество пациентов, достигших целевого уровня АД на каждом визите при лечении исследуемыми препаратами.

\section{Переносимость терапии}

Отмечено редкое возникновение побочных эффектов при лечении препаратами рамиприла. Во время исследования было зарегистрировано 4 побочных эффекта: все случаи регистрировались на фоне приема Хартила, однако 1 из них был связан с назначением Кардилопина (характерный для препаратов - антагонистов кальциевых каналов отек лодыжек). Зарегистрированные во время исследования побочные реакции перечислены в табл. 3.

Значимого различия по показателям безопасности между исследуемыми препаратами выявлено не было ( $p=0,11$; точный критерий Фишера). 
Table 2. The drugs efficacy evaluation (in accordance to visit number)

\begin{tabular}{|c|c|c|c|c|}
\hline \multirow[t]{2}{*}{ Drug } & \multicolumn{3}{|c|}{ The number of patients achieved the target BP } & \multirow{2}{*}{$\begin{array}{l}\text { The number of patients } \\
\text { not achieved the target BP }\end{array}$} \\
\hline & Visit after 2 weeks & Visit after 4 weeks & Visit after 6 weeks & \\
\hline Hartil & $0(0 \%)$ & $\begin{array}{c}+7(27 \%) \\
\text { (the total }-7(27 \%))\end{array}$ & $\begin{array}{l}+3(11,5 \%) \\
\text { (the total }-10(38,5 \%))\end{array}$ & $16(61,5 \%)$ \\
\hline Tritace & $3(11,5 \%)$ & $\begin{array}{c}+5(19,2 \%) \\
\text { (the total }-8(30,7 \%) \text { ) }\end{array}$ & $\begin{array}{c}+5(19,2 \%) \\
\text { (the total }-13(50 \%))\end{array}$ & $13(50 \%)$ \\
\hline$\chi^{2}, p$ & $\chi^{2}=2,84 ; p=0,09$ & $\chi^{2}=0,09 ; p=0,76$ & $\chi^{2}=0,70 ; p=0,4$ & $\chi^{2}=0,70 ; p=0,4$ \\
\hline
\end{tabular}

Таблица 2. Оценка эффективности препаратов (по визитам)

\begin{tabular}{|c|c|c|c|c|}
\hline \multirow[t]{2}{*}{ Препарат } & \multicolumn{3}{|c|}{ Число пациентов, достигших целевого уровня АД } & \multirow{2}{*}{$\begin{array}{c}\text { Число пациентов, не } \\
\text { достигших целевого АД }\end{array}$} \\
\hline & Визит 2 нед & Визит 4 нед & Визит 6 нед & \\
\hline Хартил & $0(0 \%)$ & $\begin{array}{c}+7(27 \%) \\
(\text { всего }-7(27 \%))\end{array}$ & $\begin{array}{c}+3(11,5 \%) \\
(\text { всего - } 10(38,5 \%))\end{array}$ & $16(61,5 \%)$ \\
\hline Тритаце & $3(11,5 \%)$ & $\begin{array}{c}+5(19,2 \%) \\
(\text { всего }-8(30,7 \%))\end{array}$ & $\begin{array}{c}+5(19,2 \%) \\
(\text { всего - } 13(50 \%))\end{array}$ & $13(50 \%)$ \\
\hline$\chi^{2}, p$ & $\chi^{2}=2,84 ; p=0,09$ & $\chi^{2}=0,09 ; p=0,76$ & $\chi^{2}=0,70 ; p=0,4$ & $\chi^{2}=0,70 ; p=0,4$ \\
\hline
\end{tabular}

Table 3. Side effects frequency

\begin{tabular}{|lccc|}
\hline Side effect & $\begin{array}{c}\text { Hartil/connection } \\
\text { with the drug }+/-\end{array}$ & $\begin{array}{c}\text { Tritace/connection with } \\
\text { the drug }+/-\end{array}$ & $\begin{array}{c}\text { Cardilopin/connection with } \\
\text { the drug }+/-\end{array}$ \\
\hline Cough, $n$ & $1 /+$ & 0 & 0 \\
\hline Allergic dermatitis, $n$ & $2 /+$ & 0 & 0 \\
\hline Ankles edema, $n$ & 0 & 0 & $1 /+$ \\
\hline The total of the cases & 3 & 0 & 1 \\
\hline
\end{tabular}

Таблица 3. Частота побочных эффектов

\begin{tabular}{|lccc|}
\hline Побочный эффект & $\begin{array}{c}\text { Хартил/ связь } \\
\text { спрепаратом +/- }\end{array}$ & $\begin{array}{c}\text { Тритаце/ связь } \\
\text { спрепаратом +/- }\end{array}$ & $\begin{array}{c}\text { Кардилопин/ связь } \\
\text { спрепаратом +/- }\end{array}$ \\
\hline Кашель & $1 /+$ & 0 & 0 \\
\hline Аллергический дерматит & $2 /+$ & 0 & 0 \\
\hline Отек лодыжек & 0 & 0 & $1 /+$ \\
\hline Всего случаев & 3 & 0 & 1 \\
\hline
\end{tabular}

ative frequencies in the groups $\left.-\chi^{2}=0,7, p=0,4\right)$. Table 2 demonstrates a number of patients (at every visit) that achieved the target BP at treatment with the studied drugs.

\section{Treatment safety}

Side effects were infrequent at ramipril treatment and were registered in only 4 patients, all the cases - at Hartil therapy, however one of them was connected with Cardilopin treatment (ankles edema). Side effects registered during the study are listed in Table 3.

There were no significant differences in safety indices between the studied drugs ( $p=0,11$; the Fisher's exact test).

\section{Обсуждение}

По результатам исследования НОРЕ, назначение ингибитора АПФ рамиприла приводит к существенному снижению исходно высокого риска сердечно-сосудистых осложнений [15]. Поэтому назначение этого препарата больным АГ 1-2-й степени с высоким риском, обусловленным сочетанием АГ с ИБС, СД, мозговым инсультом (в анамнезе) вполне оправданно. Согласно рекомендациям Европейского общества кардиологов [14], целевой уровень АД для пациентов с АГ и высоким и очень высоким риском осложнений определен ниже 130/80 мм рт.ст.

По результатам проведенного исследования, достижение целевого уровня АД у пациентов с АГ 1 -2-й степени 


\section{Discussion}

According to HOPE trial results ACE inhibitor ramipril significantly decreases initially high risk of cardiovascular complications [15]. That is why prescription of this drug to patients with $\mathrm{HT}$ of 1 -2 degree with high risk due to combination of HT with IHD, diabetes or previous stroke is fully justified. According to European Society of Cardiology guidelines [14] the target BP of hypertensive patients with high and very high risk of complications must be below $130 / 80 \mathrm{~mm} \mathrm{Hg}$.

Results of the study show that the target BP in patients with HT of 1-2 degree and high cardiovascular risk can be achieved at ramipril treatment (both original and generic) in doses not less than $10 \mathrm{mg}$ per day. However in most cases monotherapy turns out to be ineffective in such patients and does not allow reaching the target BP. Treatment with the new ramipril generic Hartil in dose of $10 \mathrm{mg} /$ day (monotherapy) resulted in the target BP achievement in less than one third of the patients $(27 \%)$, and in combination with amlodipine $5 \mathrm{mg}$ - in less than a half of the patients (38,5\%). Thus, combined therapy (ramipril and amlodipine) increases treatment efficacy.

These results are in line with a number of large clinical trials data, which showed that antihypertensive monotherapy seldom allows reaching the target BP level, especially in patients with high risk of cardiovascular complications [16-18]. So, according to ACCOMPLISH trial results, $38 \%$ of high-risk patients received three or more antihypertensive drugs at the beginning of the study, at that only $37 \%$ of the patients had BP below $140 / 90 \mathrm{~mm} \mathrm{Hg}$ [19]. That is why in accordance with European Society of Cardiology guidelines [14] combined antihypertensive therapy should be administered to patients with high cardiovascular risk if BP exceeds the target level by more than 20/10 mm Hg.

Hartil therapy demanded addition of amlodipine more often compared to Tritace treatment. Tritace was also more efficient in combined therapy with Cardilopin, which allowed to achieve the target BP in $50 \%$ of the patients. However these distinctions between both studied drugs efficacy were not significant.

\section{Conclusion}

Therapeutic equivalence of the new generic ramipril Hartil to the original drug Tritace was demonstrated in patients with $\mathrm{HT}$ of 1-2 degree and high cardiovascular risk. However efficacy of ramipril monotherapy in hypertensive patients with IHD, diabetes and previous stroke was low. Combined treatment with two antihypertensive drugs was efficient in $40-50 \%$ of cases. и высоким сердечно-сосудистым риском возможно лишь при назначении препаратов рамиприла (и оригинального, и дженерического) в дозе не ниже 10 мг в сутки. Однако в большинстве случаев монотерапия у таких пациентов оказывается недостаточно эффективной и не позволяет достичь целевого уровня АД. Лечение новым дженерическим препаратом рамиприла Хартилом в дозе 10 мг/сут (монотерапия) позволило достичь целевого уровня АД менее чем у трети больных АГ 1-2-й степени и высоким риском осложнений (27\%), а при добавлении амлодипина в дозе 5 мг - менее чем у половины пациентов (в 38,5 \% случаев). Таким образом, при добавлении к терапии рамиприлом препарата амлодипина (перевод пациента на комбинированную терапию) удается повысить эффективность проводимого лечения.

Эти результаты согласуются с данными ряда крупных клинических исследований, показывающих, что монотерапия антигипертензивными препаратами редко позволяет достичь целевого уровня АД, особенно у пациентов с высоким риском сердечно-сосудистых осложнений [16-18]. Так, по результатам исследования ACCOMPLISH, на момент включения в исследование 38\% пациентов высокого риска получали терапию тремя и более антигипертензивными препаратами, и тем не менее, только у $37 \%$ больных уровень АД был менее 140/90 мм рт.ст. [19]. Поэтому, по рекомендациям Европейского общества кардиологов [15], пациентам с высоким риском сердечнососудистых осложнений при превышении целевого уровня АД более чем на 20/10 мм рт.ст. необходимо назначение комбинированной антигипертензивной терапии.

По сравнению соригинальным препаратом Тритаце при лечении дженериком рамиприла Хартилом добавление к терапии препарата амлодипина Кардилопина требовалось в большем числе случаев. Оригинальный препарат рамиприла Тритаце был эффективен в большем числе случаев, в том числе и при комбинированной терапии с Кардилопином, в результате чего удалось достичь целевого уровня АДу 50 \% больных. Однако эти различия в эффективности исследуемых препаратов были незначимы.

\section{Заключение}

Продемонстрирована терапевтическая эквивалентность нового дженерического препарата рамиприла Хартила оригинальному препарату Тритаце у больных АГ 1-2-й степени и высоким сердечно-сосудистым риском. Однако у пациентов с АГ 1-2-й степени с высоким риском, обусловленным сочетанием АГ с ИБС, СД, мозговым инсультом, выявлена низкая эффективность монотерапии рамиприлом. Комбинированная терапия двумя антигипертензивными препаратами эффективна в $40-50 \%$ случаев. 


\section{References/Литература}

1. Kaplan NM. The CARE Study: a postmarketing evaluation of ramipril in 11,100 patients. The Clinical Altace Real-World Efficacy (CARE) Investigators. Clin Ther 1996;18(4):658-70.

2. Marre $\mathrm{M}$, Lievre $\mathrm{M}$, Chatellier $\mathrm{G}$ et al. Effects of low dose ramipril on cardiovascular and renal outcomes in patients with type 2 diabetes and raised excretion of urinary albumin: randomised, double blind, placebo controlled trial (the DIABHYCAR study). BMJ 2004;328(7438):495.

3. Dalla Vestra M, Pozza G, Mosca A. et al. Effect of lercanidipine compared with ramipril on albumin excretion rate in hypertensive Type 2 diabetic patients with microalbuminuria: DIAL study (diabete, ipertensione, albuminuria, lercanidipina). Diabetes Nutr Metab 2004;17(5):259-66.

4. Agabiti-Rosei E, Ambrosioni E, Dal Palù C et al. ACE inhibitor ramipril is more effective than the beta-blocker atenolol in reducing left ventricular mass in hypertension. Results of the RACE (RAmipril Cardioprotective Evaluation) study. J Hypertens 1995;13(11):1325-34

5. Hall AS, Murray GD, Ball SG. Follow-up study of patients randomly allocated ramipril or placebo for heart failure after myocardial infarction: AIRE Extension (AIREX) study. Lancet 1997;349:1493-7.

6. McMurray JJV, Östergren J, Swedberg K et al. Effects of candesartan in patients with chronic heart failure and reduced left-ventricular systolic function taking angiotensin-converting-enzyme inhibitors: the CHARM-Added trial. Lancet 2003;362(9386):767-71

7. Cleland JGF, Erhardt L, Murray G et al. Effect of ramipril on morbidity and mode of death among survivors of acute myocardial infarction with clinical evidence of heart failure. A report from the AIRE study investigators. Eur Heart J 1997:18(1):41-51.

8. Kjøller-Hansen L, Steffensen R, Grande P. The Angiotensin-converting enzyme inhibition Post Revascularization Study (APRES). J Am Coll Cardiol 2000;35(4):881-8

9. Pfeffer MA, Greaves SC, Arnold JM et al. Early versus delayed angiotensin-converting enzyme inhibition therapy in acute myocardial infarction. The Healing and Early Afterload Reducing Therapy trial. Circulation 1997;95(12):2643-51.

10. Yusuf S, Teo KK, Pogue J et al., ONTARGET Investigators. Telmisartan, ramipril, or both in patients at high risk for vascular events. N Engl J Med 2008;358(15):1547-59.
11. Agodoa LY, Appel L, Bakris GL et al. Effect of ramipril vs amlodipine on renal outcomes in hypertensive nephrosclerosis. JAMA 2001;285(21):2719-28.

12. Wright JT Jr, Bakris G, Greene T. et al. Effect of blood pressure lowering and antihypertensive drug class on progression of hypertensive kidney disease. JAMA 2002;288:2421-31

13. Ruggenenti P, Perna A, Gherardi $G$ et al. Renoprotective properties of ACEinhibition in non-diabetic nephropathies with non-nephrotic proteinuria. Lancet 1999;354(9176):359-64.

14. 2007 Guidelines for the management of arterial hypertension: The Task Force for the Management of Arterial Hypertension of the European Society of Hypertension (ESH) and of the European Society of Cardiology (ESC). Eur Heart J. 2007;28(12):1462-536.

15. Yusuf S, Sleight P, Pogue J et al. Effects of an angiotensin-converting-enzyme inhibitor, ramipril, on cardiovascular events in high-risk patients. The Heart Outcomes Prevention Evaluation Study Investigators. N Engl J Med 2000; 342(3):145-53.

16. Brugts JJ, Ninimiya T, Boersma YЮ et al. The consistency of the treatment ef fect of an ACE-inhibitor based treatment regimen in patients with vascular disease or high risk of vascular disease: a combined analysis of individual data of ADVANCE, EUROPA and PROGRESS trials. Eur Heart J 2009; 30(11): 1385-94.

17. Neal B., MacMahon S., Chapman N. for the Blood Pressure Lowering Treatment Trialists' Collaboration. Effects of ACE inhibitors, calcium antagonists and other blood-pressure-lowering drugs: results of prospectively designed overviews of randomized trials. Lancet 2000;355(9246):1955-64.

18. Chobanian AV. Does it matter how hypertension is controlled? N Engl I Med 2008;359(23):2485-8.

19. Jamerson KA, Bakris GL, Wun C-C et al. Rationale and design of the avoiding cardiovascular events through combination therapy in patients living with systolic hypertension (ACCOMPLISH) trial: the first randomized controlled trial to compare the clinical outcome effects of first-line combination therapies in hypertension. Am J Hypertens 2004;17(9):793-801

Received/Поступила 28.10.2009

Accepted /Принята в печать 17.11.2009 